\title{
Acupuncture Reduces Experimental Renovascular Hypertension Through Mechanisms Involving Nitric Oxide Synthases
}

\author{
DAVID D. KIM, ARNALDO M. PICA, RICARDO G. DURÁN, AND WALTER N. DURÁN \\ Program in Vascular Biology, Department of Pharmacology and Physiology, \\ UMDNJ-New Jersey Medical School, Newark, New Jersey, USA
}

\begin{abstract}
Objective: To test the hypothesis that acupuncture on stomach 36 point (ST-36) reduces hypertension by activating nitric oxide synthase signaling mechanisms.

Methods: The authors used the two-kidney, one-clip renal hypertension (2K1C) hamster model with electroacupuncture treatment.

Results: Thirty-minute daily electroacupuncture treatment for 5 days reduced mean arterial pressure from $160.0 \pm 7.6$ to $128.0 \pm 4.3 \mathrm{mmHg}$ (mean $\pm \mathrm{SEM}$ ), compared to $115.0 \pm 7.2 \mathrm{mmHg}$ in sham-operated hamsters. Electroacupuncture increased periarteriolar NO concentration from $309.0 \pm$ $21.7 \mathrm{nM}$ to $417.9 \pm 20.9 \mathrm{nM}$ in the $2 \mathrm{~K} 1 \mathrm{C}$ hamster cheek pouch microcirculation when measured with NO-sensitive microelectrodes. Hypertension reduced endothelial nitric oxide synthase (eNOS) and neuronal nitric oxide synthase (nNOS) proteins relative to the sham-operated control, as measured by Western blotting. Electroacupuncture prevented the reduction of eNOS and nNOS associated with hypertension and showed even higher eNOS and nNOS expressions than sham-operated control in stomach and cheek pouch tissues, which are on the stomach meridian. Analysis of liver tissue, a nonstomach-meridian organ, indicated that electroacupuncture did not have a significant benefit in terms of enhanced expressions of eNOS and nNOS in the treated $2 \mathrm{~K} 1 \mathrm{C}$ hypertensive group.
\end{abstract}

Conclusions: Activation of eNOS and nNOS is one of the mechanisms through which ST-36 electroacupuncture reduces blood pressure; this reduction works through the stomach meridian.

Microcirculation (2006) 13, 577-585. doi:10.1080/10739680600885210

KEY WORDS: acupuncture, complementary and alternative medicine, hypertension, meridian theory, microcirculation, nitric oxide, nitric oxide synthase, ST-36

Complementary and alternative medicine (CAM), which includes acupuncture and herbal medicine, is a current focus of interest for the general public and the medical profession. Acupuncture and herbal medicine have been used for a long time in the treatment of cardiovascular disease in Asia. In particular, acupuncture treatment has been used traditionally for the reduction of hypertension. However, this ancient practice has not yet been subjected systematically and thoroughly to the rigors of scientific testing.

This work was supported by grant 5RO1 HL 70634 from the National Institutes of Health.

Address correspondence to David D. Kim, PhD, Department of Pharmacology and Physiology, UMDNJ-New Jersey Medical School, 185 South Orange Avenue, MSB H-661, Newark, NJ 07101-1709,USA.E-mail: kimda@umdnj.edu

Received 11 February 2006; accepted 18 May 2006.
Hypertension affects millions of people in the United States and in the world. It causes loss of lives and carries significant economic cost. In addition, some medical therapeutic regimens and medications available for treating hypertension have significant side effects. Thus, it is important to investigate the mechanisms of action responsible for the benefits of acupuncture in the treatment of hypertension. The scientific assessment of this alternative treatment modality may lead to greater success in curing or controlling this silent killer, and to minimization of the side effects of some medical therapeutic regimens and medications.

The vascular regulatory site for the control of blood pressure by adjustments of total peripheral resistance resides mainly in arterioles, the precapillary segment of the microvasculature $[21,32,41]$. The pathogenesis of hypertension has been associated with the function of endothelial nitric oxide synthase (eNOS), 
an enzyme that plays a crucial role in the regulation of vessel diameter, which is an important determinant of blood flow and resistance $[1,13,22]$. Endothelial nitric oxide synthase catalyzes the production of nitric oxide ( $\mathrm{NO}$ ), which in turn mediates arteriolar vasodilatation, reduces peripheral resistance, and lowers the blood pressure necessary to bring normal blood flow to tissues. Thus, alterations in the function of this enzyme can lead to elevation of blood pressure. In fact, experimental animals lacking this enzyme demonstrate hypertension $[12,18$, $38]$.

Ma et al. demonstrated that electroacupuncture on stomach 36 point (ST-36) induces upregulation of neuronal nitric oxide synthase (nNOS) expression in the gracile nucleus and medial nucleus tractus solitarius, and this enhanced nNOS-NO in the nuclei may modify central cardiovascular regulation $[4,24,26]$. Therefore, we also investigated the impact of acupuncture on nNOS activity in normal and renovascular hypertensive hamsters, inasmuch as eNOS and nNOS are present in the hamster cheek pouch [37].

We hypothesized that acupuncture may reduce hypertension by activation of microvascular nitric oxide synthase (NOS). Among the acupuncture points, ST-36 has been used to promote blood flow and to treat cardiovascular disease [5, 10, 23, 24, 26, 44]. Thus, we used ST-36 to investigate the hypothesis that acupuncture induces its beneficial antihypertensive effects by activating NOS in the microcirculation.

\section{MATERIALS AND METHODS}

The experimental protocols for hamsters were approved by the UMDNJ-New Jersey Medical School's Institutional Animal Care and Use Committee and conducted in accordance with the NIH Guidelines for the Use of Animals.

\section{Induction of Hypertension}

We applied the two-kidney, one-clip (2K1C) protocol to induce renal hypertension in male golden Syrian hamsters, weighing $80-120 \mathrm{~g}$. Animals were anesthetized with sodium pentobarbital $(50 \mathrm{mg} / \mathrm{kg}$ IP). We used a retroperitoneal approach and placed a sliver clip (3-mm width, 5-mm length, 0.23-mm inner space) to constrict the right renal artery. The renal vein and ureter were uncompromised by the clip. After surgery, muscle lavers and skin were sutured. Sham-operated hamsters underwent the same proce- dure, except for placement of a clip. Buprenorphine $(0.1 \mathrm{mg} / \mathrm{kg} \mathrm{SC})$ was administered upon recovery and $24 \mathrm{~h}$ later to prevent pain. Hamsters were caged and maintained until their use in experiments 2 weeks later.

\section{Hamster Cheek Pouch Preparation}

Hamsters were anesthetized with sodium pentobarbital $(50 \mathrm{mg} / \mathrm{kg}$ IP). Tracheotomy was performed to ensure a clear airway passage. The right jugular vein was cannulated for administration of supplemental doses of anesthetic. The right carotid artery was cannulated for monitoring blood pressure using a PowerLab Pressure Monitor (AD Instruments, Colorado Springs, CO). The left hamster cheek pouch was prepared for intravital microscopy as described previously $[7,8,15]$.

\section{Periarteriolar NO Measurement}

The NO concentration was measured with a NOsensitive microelectrode $[2,29]$. The microelectrodes were polarized at $+0.9 \mathrm{~V}$ and the current generated was measured with an electrometer (model 6517A, Keithley, Cleveland, OH). Calibration, using a gas tonometer at $37^{\circ} \mathrm{C}$, was performed for each experiment by measuring the microelectrode current generated by 0,600 , and 1,200 nM NO. Microelectrodes having a linear relationship of electrical current to NO concentration were used. After the microelectrode tip was properly located on the arteriolar wall, the tissue and microelectrode were allowed to stabilize for $1 \mathrm{~h}$ before experimental protocols were implemented.

\section{Experimental Protocol}

Male golden Syrian hamsters were randomly divided into 4 groups: $2 \mathrm{~K} 1 \mathrm{C}$ hypertension hamsters with acupuncture, $2 \mathrm{~K} 1 \mathrm{C}$ hypertension hamsters without acupuncture, sham-operated hamsters with acupuncture, and sham-operated hamsters without acupuncture. Two weeks after the surgical operation, hamsters in the acupuncture treatment groups received acupuncture treatment of $30 \mathrm{~min}$ daily for 5 days on the ST-36 point. The ST-36 point is located on the outside of the hind leg, just below the knee, and outside of the tibial crest. The point is in the middle of the cranial tibial muscle belly $[5,6,10,23,24,26$, $35,40,44]$. A disposable sterilized acupuncture needle (diameter $0.2 \mathrm{~mm}$ ) was used. After being inserted at a depth of about 2-4 mm at ST-36 bilaterally, 
the needles were connected with an electrostimulator (model KWD-808II. Shen Zhen Kuanyu Electronic, Shen Zhen, China). The point was stimulated at $2 \mathrm{~Hz}$ with an electrical current of continuous wave at $1-\mathrm{V}$ intensity and $0.5-\mathrm{ms}$ pulse duration for $30 \mathrm{~min}$. During the treatment, the hamster was restricted in a cage (Ballman cage; Natsume Seisakusho, Tokyo, Japan). This cage has been used for light restraint in several studies $[16,28,30,39,40]$. Three days after arriving from the vendor, the hamsters were trained in the restraining cage for at least 30 min every day for a week until the day of acupuncture treatment. There is no difference in results of gastric motility between freely moving and restrained rats [40].

After the treatment, the left cheek pouch was prepared for direct visual observation and intervention according to methods described previously $[7,8,15]$. The right carotid artery was cannulated for continuous direct measurement of mean arterial blood pressure using a PowerLab Pressure Monitor. After the equilibration period, we measured the production of $\mathrm{NO}$ in each group with NO-sensitive electrodes. At the end of the experiments, the left cheek pouch, stomach, and liver were quickly dissected and excised for the quantification of changes in eNOS and nNOS proteins by Western blotting.

\section{Western Blotting Analysis}

After extractions of proteins, $80 \mu \mathrm{g}$ of protein for each sample was loaded into $7.5 \%$ polyacrylamide gels, separated by SDS-polyacrylamide electrophoresis, and transferred to polyvinylidene difluoride (PVDF) membranes (Bio-Rad, Hercules, CA) by electrophoretic elution. The membranes were incubated with mouse anti-nitric oxide synthase antibodies (BD Transduction Laboratories, San Jose, CA) that recognized human eNOS or nNOS. Subsequently, the membranes were allowed to react with mouse IgG secondary antibodies (Sigma Chemicals, St. Louis, MO). Bands corresponding to eNOS or nNOS protein were visualized using ECL system (Pierce, Rockford, IL) and analyzed with a gel documentation system (IS-1000 Digital Imaging System. Alpha Innotech, San Leandro, CA).

\section{Microscopy}

Observations were made with an Olympus BH microscope. The recording system comprises a Dage MTI 66 silicon-intensified target television camera, a Sony monitor, and a MetaMorph image system (Universal
Imaging, Downingtown, PA) for computer recording directly from the television camera and for image processing.

\section{Statistical Analysis}

All data are expressed as means \pm SEM. Statistical analysis was performed using a one-way analysis of variance. When significant values were obtained, the Student-Newmann-Keuls test was applied to determine which measurements differed significantly from another. Differences were considered significant for values of $p<.05$. All statistical analyses were performed using the InStat package (GraphPad, San Diego, CA).

\section{RESULTS}

\section{Electroacupuncture on ST-36 Point Significantly Reduces Blood Pressure in Hamsters}

Experimental constriction of the renal artery in hamsters increased mean arterial pressure (MAP) to $160.0 \pm 7.6 \mathrm{mmHg}$ (mean $\pm \mathrm{SEM}$ ) relative to $115.0 \pm 7.2 \mathrm{mmHg}$ in nontreated sham-operated hamsters. After 5 days of daily acupuncture treatment, treated $2 \mathrm{~K} 1 \mathrm{C}$ hamsters significantly reduced MAP from $160.0 \pm 7.6 \mathrm{mmHg}$ to $128.0 \pm 4.3 \mathrm{mmHg}$, i.e., a $20 \%$ reduction compared with MAP results of nontreated 2K1C hamsters (Figure 1).

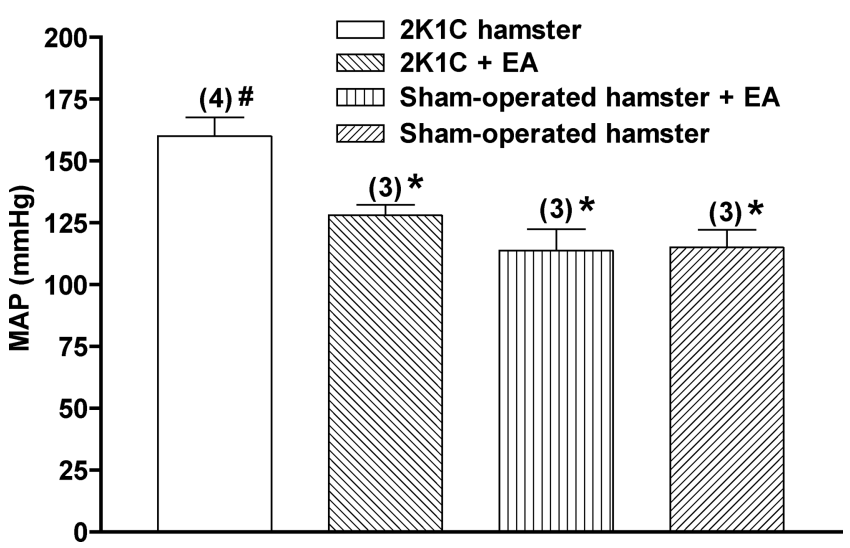

Figure 1. Electroacupuncture on ST-36 reduces mean arterial blood pressure in experimental renovascular hypertension. The bars represent the means \pm SEM. The numbers in parentheses show the number of hamsters included in each group. EA, electroacupuncture. ${ }^{*} p<.05$ compared with nontreated renovascular hypertensive $(2 \mathrm{~K} 1 \mathrm{C})$ hamsters, \#p $<.05$ compared with shamoperated hamsters. 
To investigate the effect of ST-36 point on normotensive hamster, we performed acupuncture treatment on the sham-operated hamster group. There was no significant mean arterial blood pressure difference between sham-operated hamsters with acupuncture treatments and those without acupuncture treatments.

\section{Electroacupuncture on ST-36 Increases Periarteriolar NO Concentration in Hamster Cheek Pouch Microcirculation}

To test our hypothesis, we investigated the impact of acupuncture on the production of microvascular NO in normal and renovascular hypertensive hamsters. We measured periarteriolar NO concentration with $\mathrm{NO}$-sensitive microelectrodes. Our laboratory's previous experience demonstrates that, 3 weeks after 2K1C surgery, hypertension causes a decrease in luminal diameter from $25.01 \pm 6.03 \mu \mathrm{m}$ in shamoperated hamsters to $21.39 \pm 6.39 \mu \mathrm{m}$ in the same order arterioles in $2 \mathrm{~K} 1 \mathrm{C}$ hamsters (mean $\pm \mathrm{SD}$; $p<.05$ ) [3]. To avoid potentially confounding problems of reduced diameter and endothelial mass (eNOS content), we chose to measure perivascular NO concentration in arterioles of the same luminal diameter in normo- and hypertensive animals. The mean diameter of the selected test arterioles was $38 \pm 3 \mu \mathrm{m}$.

Figure 2 shows that treated $2 \mathrm{~K} 1 \mathrm{C}$, sham-operated and treated sham-operated hamsters demonstrated a periarteriolar $\mathrm{NO}$ concentration ranging from $417.9 \pm 20.9 \mathrm{nM}$ to $440.2 \pm 31.5 \mathrm{nM}$. In contrast, nontreated $2 \mathrm{~K} 1 \mathrm{C}$ hamsters had a periarteriolar NO concentration of $309.0 \pm 21.7 \mathrm{nM}(p<.05)$. These data support the concept that electroacupuncture on ST-36 can restore the bioavailability of NO in arterioles.

Electroacupuncture on ST-36 Increases the Levels of eNOS Protein in Hamster Cheek Pouch and Stomach, Two Organs on the Stomach Meridian

We showed that acupuncture treatment on ST-36 has antihypertensive effects. Subsequently, we tested the hypothesis that these effects are regulated through eNOS activity on the stomach meridian. We determined the expression of eNOS protein in hamster cheek pouch and stomach, organs on the stomach meridian, by Western blotting (Figure 3). We detected eNOS in all 4 groups in both hamster cheek pouch and stomach and measured their respective band intensity. To standardize the anal-

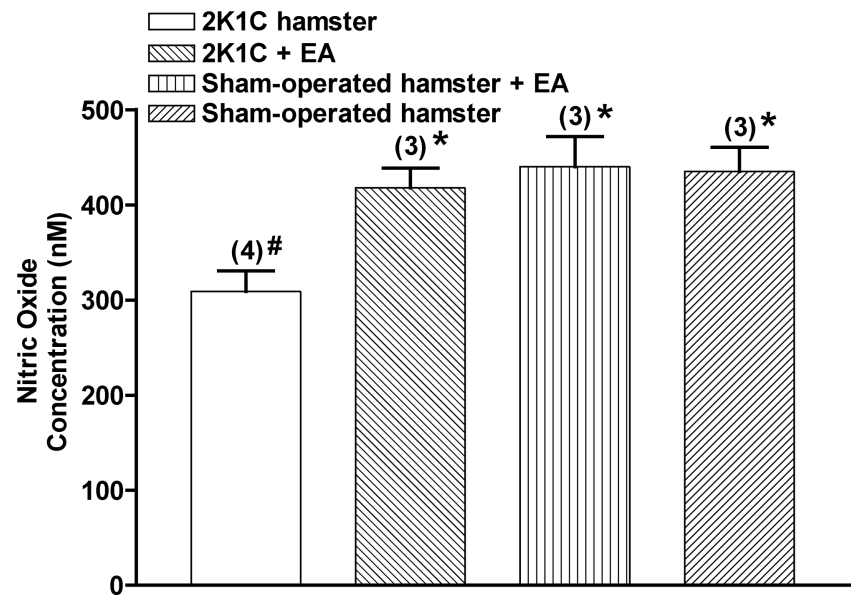

Figure 2. Electroacupuncture on ST-36 increases periarteriolar NO concentration. The bar graph shows the maximal increments in response to treatment. The data represent the means \pm SEM. The numbers in parentheses show the number of animals included in each group. EA, electroacupuncture. ${ }^{*} p<.05$ compared with nontreated $2 \mathrm{~K} 1 \mathrm{C}$ hamsters, $\# p<.05$ compared with sham-operated hamsters.

ysis, we expressed the results as the ratio of the intensity of the experimental to the corresponding sham-operated control group (i.e., 2K1C/sham). The results of the analysis of the net band intensities yielded $0.85 \pm 0.04$ (2K1C hypertensive group), $1.21 \pm 0.06$ (treated 2K1C group), and $1.06 \pm 0.09$ (treated sham-operated group) relative to the nontreated sham-operated control group in the hamster cheek pouch. The respective values in the hamster stomach were $0.77 \pm 0.04,1.05 \pm 0.15$, and $1.08 \pm 0.07$. Hypertension reduced the expression of eNOS relative to the sham-operated group. Acupuncture treatment prevented the reduction of eNOS associated with hypertension and showed even higher eNOS expression than the sham-operated control in hamster cheek pouch and stomach.

\section{Electroacupuncture on ST-36 Increases the Levels of} nNOS Protein in Hamster Cheek Pouch and Stomach

We also hypothesized that antihypertensive effects of ST-36 are regulated through nNOS activity on the stomach meridian. We examined the expression of nNOS protein in the hamster cheek pouch and stomach by Western blotting, using the same method as for eNOS to standardize the results.

Figure 4 shows the comparison of band intensity. Compared to the nontreated sham-operated group (denominator), the intensity ratios were $0.70 \pm 0.04$ 


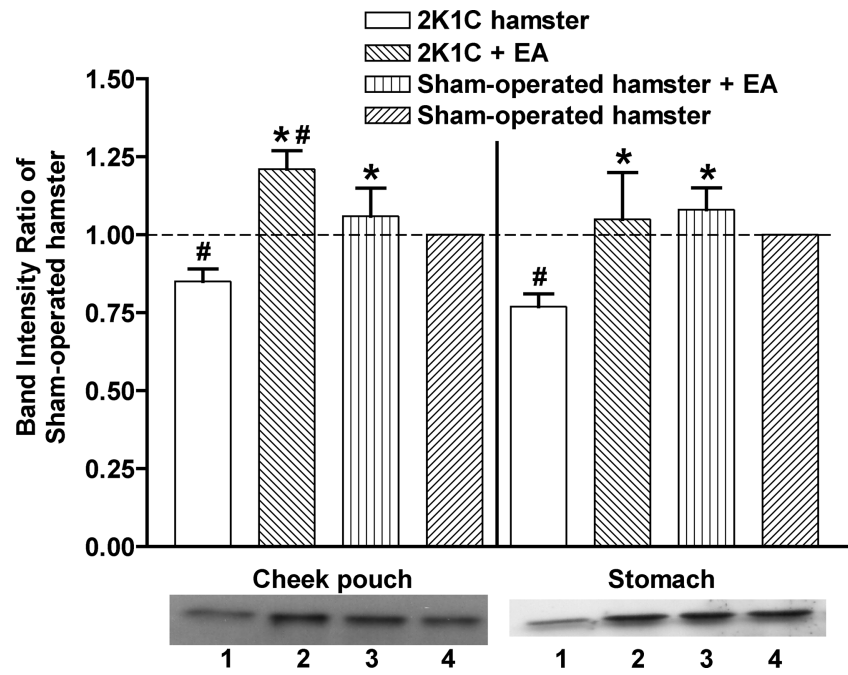

Figure 3. Western blotting analysis of eNOS in hamster cheek pouch and stomach. The band intensities in the Western blots were divided by the band intensity of corresponding nontreated sham-operated hamster. The data represent the means \pm SEM. Each group represents 3 hamsters. EA, electroacupuncture. ${ }^{*} p<.05$ compared with nontreated 2K1C hamsters, \#p $<0.05$ compared with sham-operated hamsters. The lower panel shows representative Western blot bands for each group. 1: 2K1C, 2: 2K1C+EA, 3: sham-operated+EA, 4: sham-operated.

(2K1C group), $0.85 \pm 0.03$ (treated $2 \mathrm{~K} 1 \mathrm{C}$ group), and $1.05 \pm 0.01$ (treated sham-operated group) in the hamster cheek pouch. The respective values in the stomach were $0.88 \pm 0.03,1.00 \pm 0.01$, and $1.06 \pm 0.05$. Hypertension reduced nNOS expression relative to sham-operated hamsters. Electroacupuncture treatment prevented or restored the reduction of nNOS associated with hypertension.

\section{Electroacupuncture on ST-36 Did Not Change Significantly the Levels of eNOS and nNOS Proteins in Liver, an Organ That Is Not on the Stomach Meridian}

We showed that electroacupuncture on ST-36 is regulated through eNOS and nNOS activities on the stomach meridian. To confirm the meridian theory, the expressions of eNOS and nNOS proteins in liver, which is not on the stomach meridian, were examined by Western blotting.

Figure 5 shows the comparisons of the net band intensities in liver. For eNOS, the ratios (relative to nontreated sham-operated control group) were $0.82 \pm 0.04(2 \mathrm{~K} 1 \mathrm{C}$ group), $0.85 \pm 0.04$ (treated $2 \mathrm{~K} 1 \mathrm{C}$ group), and $1.01 \pm 0.05$ (treated sham- operated group). For $\mathrm{nNOS}$ the respective values were $0.87 \pm 0.03,0.89 \pm 0.02$, and $1.01 \pm 0.03$. Analysis of liver tissue indicated that electroacupuncture on ST-36 did not have a significant benefit in terms of enhanced expression of eNOS and nNOS expressions in the treated 2K1C hypertensive group relative to the nontreated $2 \mathrm{~K} 1 \mathrm{C}$ hypertensive group. The liver eNOS and nNOS levels in nontreated $2 \mathrm{~K} 1 \mathrm{C}$ hamsters were significantly reduced relative to shamoperated hamsters.

\section{DISCUSSION}

Our study demonstrates in hamsters that electroacupuncture on stomach 36 point (1) reduces experimental renovascular hypertension, (2) increases the production of $\mathrm{NO}$ in arterioles, and (3) increases the expression of eNOS and nNOS, which serve as target signaling molecules in organs on the stomach meridian. ST-36 is one of the frequently used acupuncture points. ST-36, alone and in combination with other acupuncture points, has been used traditionally for the treatment of hypertension in Asia $[6,43]$. ST-36 combined with 3 other acupuncture points reduces diastolic blood pressure in human

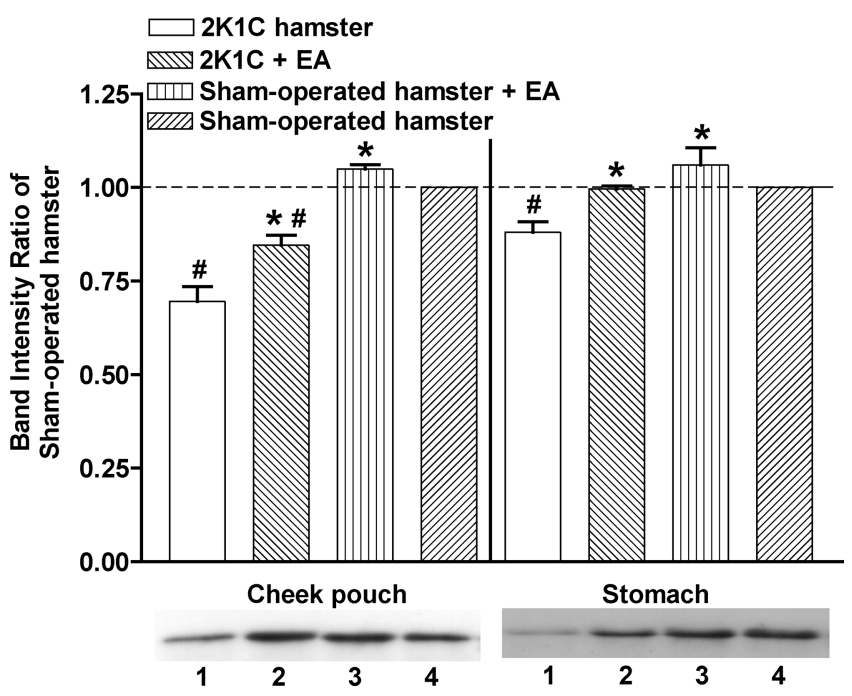

Figure 4. Western blotting analysis of nNOS in hamster cheek pouch and stomach. The band intensities in the Western blots were divided by the band intensity of corresponding nontreated sham-operated hamster. The data represent the means \pm SEM. Each group represents 3 hamsters. EA, electroacupuncture. ${ }^{*} p<.05$ compared with nontreated $2 \mathrm{~K} 1 \mathrm{C}$ hamsters, \#p<.05 compared with sham-operated hamsters. The lower panel shows representative Western blot bands for each group. 1: 2K1C, 2: 2K1C+EA, 3: sham-operated+EA, 4: sham-operated. 


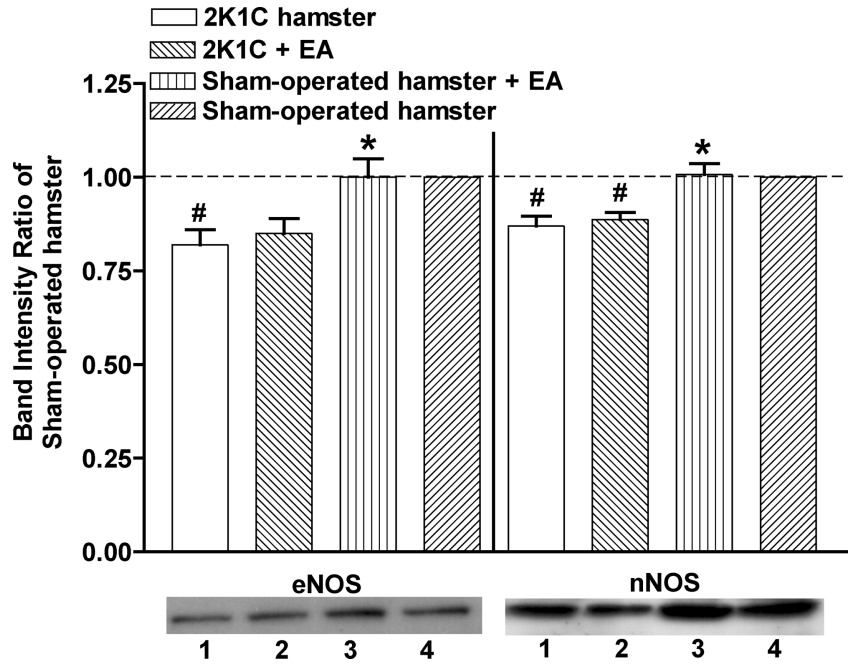

Figure 5. Western blotting analysis of eNOS and nNOS in liver. The band intensities from the Western blots were divided by the band intensity of corresponding nontreated sham-operated hamster. The data represent the means \pm SEM. Each group represents 3 hamsters. EA, electroacupuncture. ${ }^{*} p<.05$ compared with nontreated $2 \mathrm{~K} 1 \mathrm{C}$ hamsters, \# $p<.05$ compared with sham-operated hamsters. The lower panel shows representative Western blot bands for each group. 1: 2K1C, 2: 2K1C+EA, 3: sham-operated+EA, 4: sham-operated.

[42], while ST-36 in combination with large intestine (LI)-11, liver-3, and LI-4 points reduced mean arterial blood pressure in rats [45]. Our data demonstrate significant reduction of mean arterial blood pressure in renal hypertensive hamsters with ST-36 point alone. We further provide evidence that this blood pressure-lowering action is possibly mediated by stimulation of nitric oxide synthases.

It is worth noting that ST-36 lowered blood pressure in experimental renovascular hypertensive hamsters but it did not influence blood pressure in shamoperated hamsters. This observation is in agreement with a report that acupuncture treatment reduced systolic blood pressure in $2 \mathrm{~K} 1 \mathrm{C}$ hypertensive rats, but not in sham-operated rats [20]. The lack of hypotensive action of acupuncture in sham-operated control animals remains an unexplained but reproducible observation.

NO plays a crucial role in the state of blood vessel vasodilation and hence blood pressure regulation $[11,22]$. In particular, endothelial NO modulates vascular processes, including blood pressure, flow distribution, platelet aggregation, platelet and leukocyte adhesion to the endothelium, vascular smooth muscle cell proliferation, and angiogenesis [9, 17,
$31,34,36]$. Because of the important role of $\mathrm{NO}$ in these processes, abnormalities in vascular NO production are thought to contribute to the pathogenesis of hypertension. To test this hypothesis in the context of complementary and alternative medicine, we investigated the impact of acupuncture on the production of microvascular NO in normal and renovascular hypertensive hamsters. We showed that hypertension was associated with decreased periarteriolar $\mathrm{NO}$ concentration in $2 \mathrm{~K} 1 \mathrm{C}$ hypertensive hamsters as compared to NO levels in nontreated sham-operated hamsters. In agreement with our hypothesis, electroacupuncture increased periarteriolar NO concentration in treated $2 \mathrm{~K} 1 \mathrm{C}$ hamsters. These data support the concept that electroacupuncture on ST-36 either prevents a reduction of and/or restores the bioavailability of $\mathrm{NO}$ in arterioles.

\section{Impact on Meridian Theory}

We showed that electroacupuncture on ST-36 is regulated through NOS in organs on the stomach meridian. The meridian theory is an important component system that has been described in oriental medicine for thousands of years. The meridian theory deals with physiologic regulation and pathologic changes in the human body, and it guides the diagnosis and treatment of oriental medicine in many aspects, especially in relation to acupuncture $[6,27]$.

The stomach meridian starts at the lateral side of the nose, ascends laterally along the infra-orbital ridge, and continues laterally across the cheeks. It passes through the diaphragm, enters the stomach (the organ it pertains to), and connects with the spleen and the heart, specifically through the stomach divergent channel. Next, it descends inside the abdomen, reaches the knee, and ends at the lateral side of the tip of the 2nd toe [33]. Based on this ancient stomach meridian theory, the meridian passes through not only the stomach, but also the spleen and the heart. Whether these organs are involved in the reduction of blood pressure by ST-36 acupuncture treatment needs further investigation.

We demonstrated that NO is one of the signaling molecules along the meridians, which has been also supported by other investigators. While examining the distributions of $\mathrm{NO}$ in the skin acupoints of rats, Ma showed that NO content and nNOS expression were consistently higher in the skin acupoints/meridians [25]. Later, Chen and Ma showed that L-arginine-derived $\mathrm{NO}$ synthesis appears to mediate noradrenergic function on skin sympathetic 
nerve activation, which contributes to skin electrical resistance of acupoints and meridians [4]. Therefore, along with the cited findings, our results support the meridian theory and the concept that $\mathrm{NO}$ is one of the important signaling molecules for the intercellular communication in the meridian system, especially in relation to acupuncture.

In further support of the meridian theory, our results demonstrate that stimulation of ST-36 activates specific targets on the stomach meridian (cheek pouch and stomach), but does not activate them in organs located on separate meridians (liver).

\section{Nitric Oxide Synthases and Hypertension}

We hypothesized that endothelial nitric oxide synthase, among the nitric oxide synthase isoforms, is a relevant signaling target for ST-36 because eNOS is the major source of NO production in endothelial cells, and an important regulator of blood pressure $[1,13,22]$. Indeed, deletion of the gene encoding for endothelial nitric oxide leads to hypertension in mice $[12,18,38]$. The factors that downregulate the expression of eNOS in the microvasculature of $2 \mathrm{~K} 1 \mathrm{C}$ hamsters and contribute to the development of renovascular hypertension remain to be elucidated. Our data indicate that untreated hypertensive $2 \mathrm{~K} 1 \mathrm{C}$ hamsters have reduced eNOS protein and NO production relative to normotensive and sham-operated animals. We did not pursue the time course correlation between eNOS downregulation and development of hypertension; however, our results clearly demonstrate that electroacupuncture on ST-36 performed after 2 weeks of constricting the renal artery serves to restore NO production and mean arterial pressure to normal values by enhancing eNOS protein levels. Nonetheless, it would be important to determine subsequently, in a longitudinal study, whether the beneficial effect of acupuncture on ST-36 on high blood pressure is due mainly to activation of endothelial and neuronal nitric oxide synthase or to rescuing (preserving) the enzymes from damage caused by hypertension or a combination of both processes.

Electroacupuncture on ST-36 also increases the expression of nNOS in stomach and cheek pouch of hypertensive hamsters. Our data support the ability of acupuncture on ST-36 to elevate nNOS. Acupuncture treatment elevates nNOS in gracile nucleus and medial nucleus tractus solitarius [24], and contributes to enhanced nerve control of circulation and blood pressure.
Our data are in agreement with recent publications showing a reduction of NOS in renal hypertensive animals [14, 19]. Elevation in plasma renin activity is a characteristic of renovascular hypertension in hamsters [3]. The efficacy of acupuncture in reducing plasma renin activity was demonstrated in rats [20], and it is possible that electroacupuncture on ST-36 reduces blood pressure in hamsters in part through mechanisms involving plasma renin activity. However, elucidation of the contributions of the renin-angiotensin system was beyond the scope of our inquiry.

At this time, our data provide a basis to better understand the microvascular mechanisms of action of acupuncture used in the treatment of hypertension in the practice of complementary and alternative medicine. In this context, we conclude that (1) activation of eNOS and nNOS is one of the mechanisms through which ST-36 electroacupuncture reduces blood pressure; (2) ST-36 electroacupunctureinduced reduction of blood pressure works through the stomach meridian.

\section{REFERENCES}

1. Albrecht EW, Stegeman CA, Heeringa P, Henning RH. Van Goor, H. (2003). Protective role of endothelial nitric oxide synthase. J Pathol 199:8-17.

2. Bohlen HG, Nase GP. (2000). Dependence of intestinal arteriolar regulation on flow-mediated nitric oxide formation. Am J Physiol 279:H2249-H2258.

3. Boric MP. (1985). Adaptive Microvascular Mechanisms in Renovascular Hypertension. Thesis, Newark, NJ: UMDNJ-Graduate School of Biomedical Sciences, 84-85.

4. Chen JX, Ma SX. (2005). Effects of nitric oxide and noradrenergic function on skin electric resistance of acupoints and meridians. J Altern Complement Med 11:423-431.

5. Chen S, Ma SX. (2003). Nitric oxide in the gracile nucleus mediates depressor response to acupuncture (ST36). J Neurophysiol 90:780-785.

6. Deadman P, Al-Khafaji M, Baker K. (1998). A Manual of Acupuncture. Ann Arbor, MI: Cushing Malloy, 158161.

7. Dillon PK, Durán WN. (1988). The effect of platelet activating factor on microvascular permselectivity: dose-response relationships and pathways of action in the hamster cheek pouch microcirculation. Circ Res 62:732-740.

8. Dillon PK, Ritter AB, Durán WN. (1988). Vasoconstrictor effects of platelet activating factor in the hamster cheek pouch microcirculation: dose-response relationships and pathways of action. Circ Res 62:722-731. 
9. Durán WN, Seyama A, Yoshimura K, González DR. Figueroa XF, Boric, MP. (2000). Stimulation of NO production and of eNOS phosphorylation in the microcirculation in vivo. Microvasc Res 60:104-111.

10. Ernst M, Lee MH. (1986). Sympathetic effects of manual and electrical acupuncture of the tsusanli knee point: comparison with the hoku hand point sympathetic effects. $\operatorname{Exp}$ Neurol 94:1-10.

11. Gerritsen ME. (2005). Genetic variations in vascular endothelial growth factor and endothelial nitric oxide synthase and their contributions to human disease. Microcirculation 12:129-140.

12. Huang PL, Huang Z, Mashimo H, Bloch KD, Moskowitz MA, Bevan JA, Fishman MC. (1995). Hypertension in mice lacking the gene for endothelial nitric oxide synthase. Nature 377:239-242.

13. Huang PL. (2003). Endothelial nitric oxide synthase and endothelial dysfunction. Curr Hypertens Rep 5:473-480.

14. Khattab MM. Mostafa A. Al-Shabanah O. (2005). Effects of captopril on cardiac and renal damage, and metabolic alterations in the nitric oxide-deficient hypertensive rat. Kidney Blood Press Res. 28:243-250.

15. Kim DD, Ramirez MM, Durán WN. (2000). Plateletactivating factor modulates microvascular dynamics through phospholipase $\mathrm{G}$ in the hamster cheek pouch. Microvasc Res 59:7-13.

16. Kiso T, Nagakura Y, Toya T, Matsumoto N, Tamura S, Ito H, Okada M. Yamaguchi T. (2001). Neurometer measurement of current stimulus threshold in rats. $J$ Pharmacol Exp Ther 297:352-356.

17. Kubes P, Suzuki M, Granger DN. (1991). Nitric oxide: an endogenous modulator of leukocyte adhesion. Proc Natl Acad Sci USA 88:4651-4655.

18. Kubis N, Richer C, Domergue V, Giudicelli JF, Levy BI (2002). Role of microvascular rarefaction in the increased arterial pressure in mice lacking for the endothelial nitric oxide synthase gene $\left(\mathrm{eNOS}^{-/-}\right) . J$ Hypertens 20:1581-1587.

19. Kuru O, Senturk UK, Gulkesen H, Demir N, Gunduz F. (2005). Physical training increases renal injury in rats with chronic NOS inhibition. Ren Fail 27:459-463.

20. Lee HS, Kim JY. (1994). Effects of acupuncture on blood pressure and plasma renin activity in twokidney one clip Goldblatt hypertensive rats. Am J Chinese Med 22:215-219.

21. Levy Bl, Ambrosio G, Pries AR, Struijker-Boudier HA. (2001). Microcirculation in hypertension: a new target for treatment. Circulation 104:735-740.

22. Li H, Wallerath T, Munzel T, Forstermann U. (2002). Regulation of endothelial-type NO synthase expression in pathophysiology and in response to drugs. $\mathrm{Ni}$ tric Oxide 7:149-164.

23. Li P, Rowshan K, Crisostomo M, Tjen-A-Looi SC, Longhurst JC. (2002). Effect of electroacupuncture on pressor reflex during gastric distension. Am J Physiol 283:R1335-R1345.
24. Ma SX, Ma J, Moise G, Li XY. (2005). Responses of neuronal nitric oxide synthase expression in the brainstem to electroacupuncture Zusanli (ST 36) in rats. Brain Res 1037:70-77.

25. Ma SX. (2003). Enhanced nitric oxide concentrations and expression of nitric oxide synthase in acupuncture points/meridians. J Altern Complement Med 9:207215.

26. Ma SX. (2004). Neurobiology of acupuncture: toward CAM. Evid Based Complement Alternat Med 1:41-47.

27. Maciocia G. (1989). The Foundations of Chinese Medicine, New York: Churchill Livingstone.

28. Nakada Y, Yokoyama O, Komatsu K, Kodama K, Yotsuyanagi S, Niikura S, Nagasaka Y, Namiki M. $(2000)$. Effects of aniracetam on bladder overactivity in rats with cerebral infarction. J Pharmacol Exp Ther 293:921-928.

29. Nase GP, Tuttle J, Bohlen HG. (2003). Reduced perivascular $P_{\mathrm{O}_{2}}$ increases nitric oxide release from endothelial cells. Am J Physiol 285:H507-H515.

30. Noguchi M, Ikarashi Y, Yuzurihara M, Kase Y, Takeda S, Aburada M. (2003). Significance of measured elevation of skin temperature induced by calcitonin generelated peptide in anaesthetized rats. J Pharm Pharmacol 55:1547-1552.

31. Papapetropoulos A, Garcia-Cardena G, Madri JA, Sessa WC. (1997). Nitric oxide production contributes to the angiogenic properties of vascular endothelial growth factor in human endothelial cells. J Clin Invest 100:3131-3139.

32. Prewitt RL, Rice DC, Dobrian AD. (2002). Adaptation of resistance arteries to increase in pressure. Microcirculation 9:295-304.

33. Qiu ML. (1993). Chinese Acupuncture and Moxibustion. New York: Churchill Livingston, 72-73.

34. Radomski MW, Palmer RMJ, Moncada S. (1987). The anti-aggregating properties of vascular endothelium: interactions between prostacyclin and nitric oxide. $\mathrm{Br}$ J Pharmacol 92:639-646.

35. Schwartz C. (1996). Four Paws Five Directions: A Guide to Chinese Medicine for Cats and Dogs. Berkley, CA: Celestial Arts, 13-24.

36. Scott-Burden T, Vanhoutte PM. (1994). Regulation of smooth muscle cell growth by endothelium-derived factors. Tex Heart Inst J 21:91-97.

37. Segal SS, Brett SE, Sessa WC. (1999). Codistribution of NOS and caveolin throughout peripheral vasculature and skeletal muscle of hamsters. Am J Physiol 277:H1167-H1177.

38. Shesely EG, Maeda N, Kim HS, Desai KM, Krege JH, Laubach VE. Sherman PA. Sessa WC. Smithies O. (1996). Elevated blood pressures in mice lacking endothelial nitric oxide synthase. Proc Natl Acad Sci USA 93:13176-13181.

39. Sugai GCM, Freire AO, Tabosa A, Yamamura Y, Tufik S, Eugenio L. Mello AM. (2004). Serotonin 
involvement in the electroacupuncture- and moxibustion-induced gastric emptying in rats. Physiol Behavi 82:855-861.

40. Tatewaki M, Harris M, Uemura K, Ueno T, Hoshino E, Shiotani A, Pappas TN. Takahashi T. (2003). Dual effects of acupuncture on gastric motility in conscious rats. Am J Physiol Regul Integr Comp Physiol 285:R862-R872.

41. Vicaut E. (1999). Microcirculation and arterial hypertension. Drugs 58:1-10.

42. Williams T, Muller K, Cornwall MW. (1991). Effect of acupuncture-point stimulation on diastolic blood pressure in hypertensive subjects: a preliminary study. Phys Ther 71:523-529.

43. Wu Y, Fisher W, Fratkin J. (1997). Practical Therapeutics of Traditional Chinese Medicine. Brookline, MA: Paradigm, 183-189.

44. Zhou W, Fu L-W, Tjen-A-Looi SC, Li P, Longhurst JC. (2005). Afferent mechanisms underlying stimulation modality-related modulation of acupuncture-related cardiovascular responses. J Appl Physiol 98:872-880.

45. Zhou Y, Chen Q, Hou Z, Chen Y. (1993). Experimental research on treatment of hypertension with acupuncture. J Tradit Med 13:277-280. 